\title{
Simulation toolkit for detailed analysis of prompt gamma generation in hadron therapy
}

\author{
F. Diblen, S. España, R. Van Holen, S. Vandenberghe \\ MEDISIP, Department of Electronics and Information Systems, Ghent University-IBBT-IBiTech, Ghent, Belgium
}

\section{Introduction}

Worldwide, thirty-five proton and carbon ion therapy centers are active and about 85.000 patients have been treated worldwide with particle radiotherapy, of them about 75.000 with protons. These numbers are increasing because of the benefit of the so-called Bragg peak for dose delivery. In hadron therapy, small errors in range may increase dramatically the dose delivered to healthy tissue or produce suboptimal coverage of the tumor. Therefore it is very important to develop a method to verify the delivered dose and Bragg peak location during treatment.

The interaction of proton and ion beams in the patient results in the generation of high-energy prompt gammas (picoseconds) and positrons (seconds-minutes). Recent investigations have assessed the use of prompt gamma emission produced by inelastic interactions of incident protons and target nuclei as a tool to verify the proton dose delivery and range [1]. In particular interactions, the nucleus is excited to a higher energy state and emits a single photon (prompt gamma) as it returns to ground state. Due to the very sort decay time of those processes, prompt gamma imaging has some advantages compared to other techniques employed for proton dose verification, e.g. PET imaging [2]. The immediate registration of prompt gamma events during the patient irradiation which avoids biological washout, does not extend acquisition time after irradiation and allows imaging for individual pencil beams in active scanning or different components of the spread-out Bragg peak (SOBP) in passive scattering. Although there are different systems being studied, such as Anger camera, Compton camera, Electron positron pair creation camera, there is no optimized prompt gamma imaging system exists to date.

Monte Carlo simulations provide a unique tool to understand the processes involved in prompt gamma production and detection. Therefore, they are playing an important role in the design and optimization of imaging system for dose verification [3]. The work presented here shows the first stage of our project, which consists in the development of the appropriate Monte Carlo and analysis tools and the study of the prompt gammas produced by proton beams. This tool will be useful for optimizing imaging system.

\section{Methods}

A new Monte Carlo tool based on Geant4 simulation toolkit [4] is being developed that can provide very detailed information about the physics processes involved in proton irradiation. The setup employed in the simulation consist of a cylinder with average soft tissue composition $(63 \% \mathrm{O}, 23 \% \mathrm{C}, 10 \% \mathrm{H}, 2 \% \mathrm{~N}$ and density $=1.0 \mathrm{~g} / \mathrm{cm}^{3}$ ) with $40 \mathrm{~cm}$ diameter and $40 \mathrm{~cm}$ height. Proton beams with $1 \mathrm{~mm}$ FHWM transverse width and different energies (between $100 \mathrm{MeV}$ and $230 \mathrm{MeV}$ ) were generated to irradiate phantom at the center and perpendicularly to one of the bases of the cylinder. Primary and secondary particle information (position, energy, physics process etc.) were recorded in data files for $10^{6}$ incident protons with energies ranging from $100 \mathrm{MeV}$ to $230 \mathrm{MeV}$, although only results for $150 \mathrm{MeV}$ are reported here. Spectra of emitted prompt gamma particles as well as depth emission profiles were obtained. Those plots were filtered using different constraints to better understand the processes involved in the production of prompt gamma rays produced by proton beams. In first place, the plots were filtered by energy of the prompt gamma particles to study which energy produce better correlation with dose distributions. The energy ranges employed were $\mathrm{E} \gamma<1 \mathrm{MeV}, 1 \mathrm{MeV}<\mathrm{E} \gamma<3 \mathrm{MeV}, 3 \mathrm{MeV}<\mathrm{E} \gamma<10 \mathrm{MeV}$ and $\mathrm{E} \gamma>10 \mathrm{MeV}$. A second filter was the type of parent particle that led to the production of the prompt gamma, differentiating among proton, electron, positron and neutron. Finally, a filter by type of interaction that produced the prompt gamma is presented splitting profiles by inelastic, bremsstrahlung, neutron capture and annihilation.

\section{Results}

Figure 1 shows the results obtained of the prompt gamma produced by a $150 \mathrm{MeV}$ proton beam in the soft tissue phantom. Figure 1 shows the energy spectra $(d, e)$ and depth profiles $(a, b, c)$ of produced prompt gammas differentiating between range of energy (a), type of particles (b) and type of interactions (c) that produce them. Energy spectra are in very good agreement with those found in the literature [5]. As shown in [5], depth profiles show the best correlation with the dose profile for higher energy photons $(3 \mathrm{MeV}<$ 
$\mathrm{E} \gamma<10 \mathrm{MeV}$ ). Prompt gammas produced by protons are well correlated to the dose profile while neutrons and electrons show no clear peak. Prompt gammas produced by electrons can be mostly filtered by selecting energies greater than $1 \mathrm{MeV}$ while the gammas produced by neutrons have an energy similar to those produced by protons. Inelastic interactions are dominant in the production of prompt gammas and show good correlation with dose profile. Bremsstrahlung and neutron capture contributes to the background with no information of the distal falloff position.

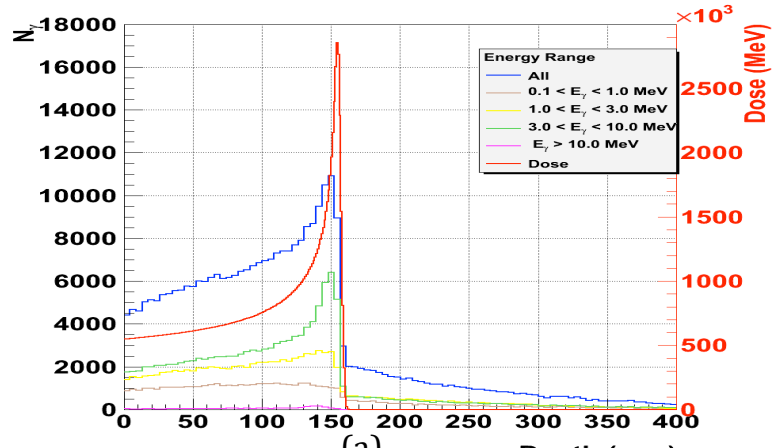

(a)

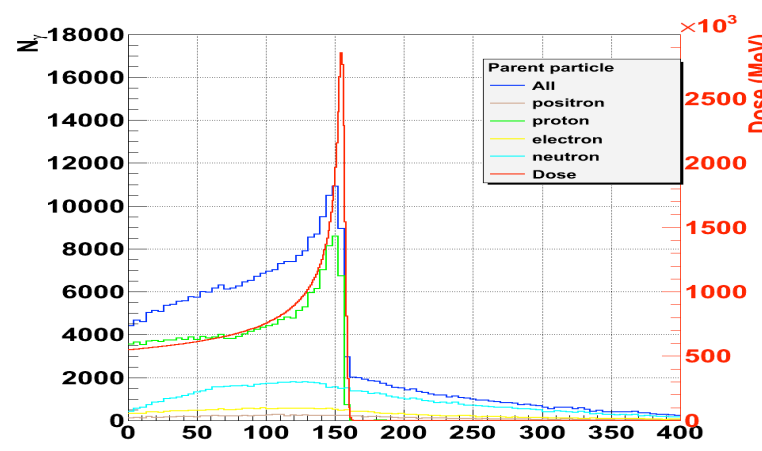

(b)

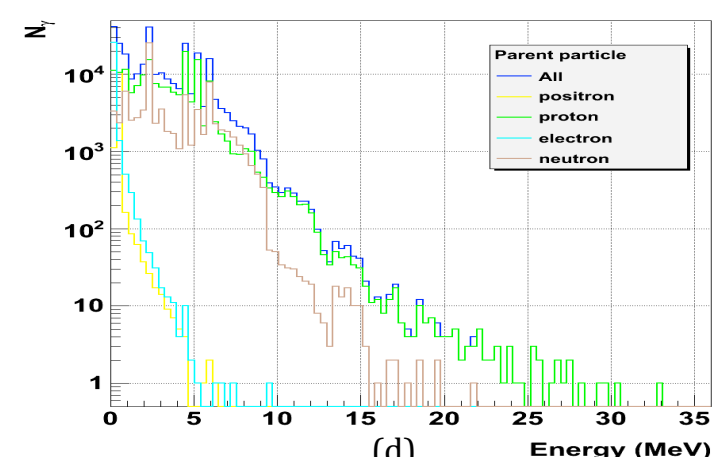

(d)

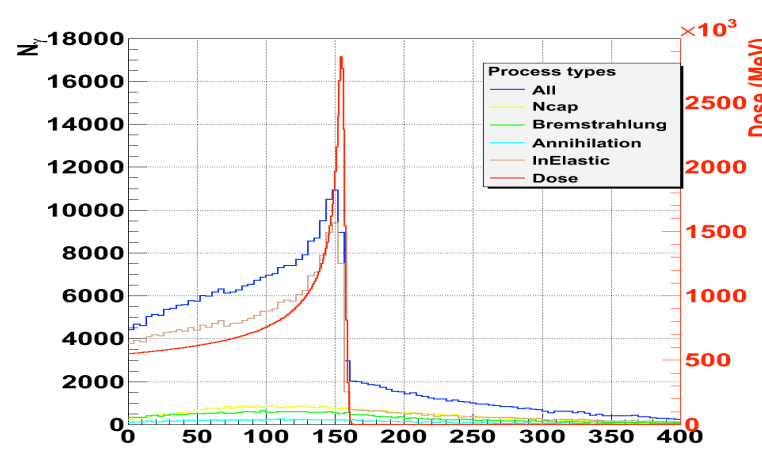

(c)

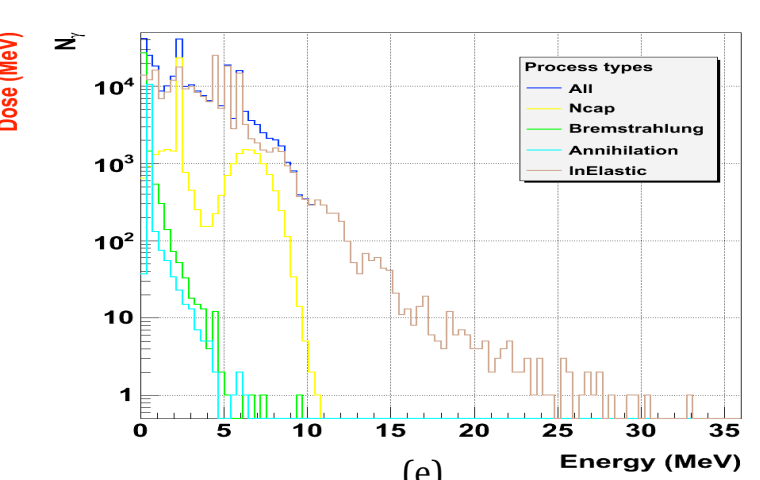

(e)

Figure 1. Depth profiles $(\mathrm{a}, \mathrm{b}, \mathrm{c})$ and energy spectra $(\mathrm{d}, \mathrm{e})$ of prompt gamma photons produced by a proton beam of $150 \mathrm{MeV}$ in a soft tissue phantom. Prompt gamma profiles for different emission energy ranges (a), type of parent particle (b) and type of interaction (c) (Ncap = neutron capture) are shown. The dose profile is also shown in depth profiles figures.

\section{Conclusion}

A Monte Carlo tool for the simulation of proton and ion beams with detailed analysis capabilities is under development. The ultimate goal of this project is to optimize the design of an imaging system for detecting prompt gamma photons to verify dose and determine Bragg peak location in proton and ion beam therapy. A detailed study of the prompt gamma emission process was presented here that will guide the future work. A validation of simulation will be accomplished.

\section{References}

[1] Polf J C et al 2009 Phys. Med. Biol. 54 731-43

[2] Enghardt W et al 2004 Nucl. Instr. Meth. Phys. Res. A 525 284-88

[3] Richard M H 2011 IEEE Trans. Nuc. Sci. 58, 87-94

[4] Agostinelli S et al 2003 Nucl. Instrum. Methods. Phys. Res. A 506 250-303

[5] Kim D et al 2009 J. Korean Phys. Socie. 55 1673-6 